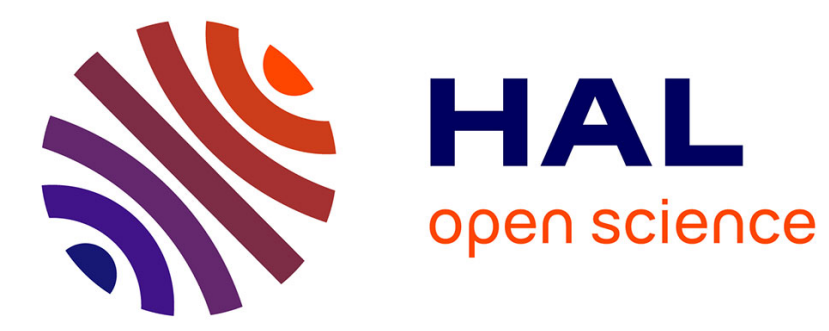

\title{
Nielsen-Thurston orderings and the space of braid orderings
}

\author{
Andres Navas, Bert Wiest
}

\section{To cite this version:}

Andres Navas, Bert Wiest. Nielsen-Thurston orderings and the space of braid orderings. Bulletin of the London Mathematical Society, 2011, 43 (5), pp.901-911. 10.1112/blms/bdr027 . hal-00395102

\section{HAL Id: hal-00395102 \\ https://hal.science/hal-00395102}

Submitted on 14 Jun 2009

HAL is a multi-disciplinary open access archive for the deposit and dissemination of scientific research documents, whether they are published or not. The documents may come from teaching and research institutions in France or abroad, or from public or private research centers.
L'archive ouverte pluridisciplinaire HAL, est destinée au dépôt et à la diffusion de documents scientifiques de niveau recherche, publiés ou non, émanant des établissements d'enseignement et de recherche français ou étrangers, des laboratoires publics ou privés. 


\title{
NIELSEN-THURSTON ORDERINGS AND THE SPACE OF BRAID ORDERINGS
}

\author{
ANDRÉS NAVAS AND BERT WIEST
}

\begin{abstract}
We study the topological space of left-orderings of the braid group, and its subspace of Nielsen-Thurston orderings. Our main result is that no Nielsen-Thurston ordering is isolated in the space of braid orderings. In the course of the proof, we classify the convex subgroups and calculate the Conradian soul for any Nielsen-Thurston ordering of $B_{n}$. We also prove that for a large class of Nielsen-Thurston orderings, including all those of infinite type, a stronger result holds: they are approximated by their own conjugates. On the other hand, we suggest an example of a Nielsen-Thurston ordering which may not be approximated by its conjugates.
\end{abstract}

\section{INTRODUCTION}

In recent years, some progress in the theory of orderable groups has been achieved by studying the so-called space of orderings (throughout, the word ordering stands for a left-invariant total order relation on a group). This corresponds to the set of all orderings that may be defined on a given group $\Gamma$, and carries a natural topology: given finitely many group elements, a neighborhood of a prescribed ordering $\prec$ is the set of all orderings which coincide with $\prec$ on this finite set. It is easy to see that this space is totally disconnected and compact; moreover, it is metrizable when the underlying group is countable. It is a nontrivial fact that this space cannot be countably infinite $[7,8,9$. Notice that an isolated point in this space corresponds to an ordering which is completely determined by finitely many inequalities.

Remarkable examples of orderable groups are the braid groups $B_{n}$. Historically, the first ordering on $B_{n}$ (for $n \geqslant 3$ ) was defined by Dehornoy. It was shown in [8] that the Dehornoy ordering $\prec_{D}$ is non isolated in the space of braid orderings. This is to be contrasted with the work [3], where Dubrovina and Dubrovin show, by means of a slight modification of the Dehornoy construction, that $B_{n}$ admits isolated orderings. For example, on $B_{3}$ there is a unique ordering $\prec_{D D}$ verifying the inequalities $\sigma_{1} \sigma_{2} \succ_{D D} 1$ and $\sigma_{2}^{-1} \succ_{D D} 1$.

Dehornoy's construction involves some deep ideas which are purely algebraic. However, there is an alternative geometric approach to braid orderability. Indeed, $\prec_{D}$ can be seen as a particular member of a family of uncountably many orderings, each of which is associated to a completely separating geodesic of the punctured disk (endowed with a hyperbolic metric). These so-called Nielsen-Thurston orderings on braid groups where largely studied and classified by H. Short and the second-named

2000 Mathematics Subject Classification. 20F36, 20F60; 06F15. 
author in [10]. In the present paper we pursue this study from the point of view of the space of orderings. Our main result may be stated as follows.

Theorem. No Nielsen-Thurston ordering is isolated in the space of braid-orderings.

We recall that the positive cone of an ordering is the semigroup consisting of elements greater than the identity. As a direct consequence of this theorem and 8 , Proposition 1.8] we have:

Corollary. There is no Nielsen-Thurston ordering whose positive cone is finitely generated as a semigroup.

To show the theorem, we first determine the convex subgroups of Nielsen-Thurston orderings (where a subset $S$ is said to be convex if $g \in S$ whenever $f_{1} \prec g \prec f_{2}$ and both $f_{1}, f_{2}$ are in $S$ ). We prove that the only convex subgroups are the obvious ones, coming from the way in which the geodesic cuts up the surface. For example, for the Nielsen-Thurston orderings of full infinite type (to be defined below), we prove that they have no convex subgroups other than $\{1\}$ and $B_{n}$. This solves by the affirmative the second half of Question 2.21 in [2, Chapter XVI].

With the whole list of convex subgroups at hand, for each Nielsen-Thurston ordering we determine the Conradian soul, that is the maximal convex subgroup restricted to which the ordering satisfies the so-called Conrad property. This notion was introduced by the first-named author as a tool for studying the possibility of approximating a given ordering by its conjugates (where the conjugate of an ordering $\prec$ by a group element $h$ is the ordering $\prec_{h}$ for which $f \prec_{h} g$ if and only if $\left.h^{-1} f h \prec h^{-1} g h\right)$. For example, by [8] every ordering on a countable group having trivial Conradian soul may be approximated by its conjugates. As a consequence, Nielsen-Thurston orderings of full infinite type can be approximated by their conjugates.

For Nielsen-Thurston orderings whose Conradian soul is isomorphic to $\mathbb{Z}$ (as is the case of the Dehornoy ordering), we show that the property of accumulation by its conjugates still holds. In the rest of the cases, we show that the Conradian soul is isomorphic to $\mathbb{Z}^{k}$ for some $k \geqslant 2$. Although we ignore whether these orderings may be approximated by their conjugates (actually, we present evidence that some of them might be isolated in the "space of Nielsen-Thurston orderings"), we show that they are not isolated in the space of braid orderings. This is achieved by means of a classical and very simple convex extension type argument.

\section{A BRIEF REMINDER ON NiELSEN-ThURSTON ORDERINGS}

Nielsen-Thurston orderings of $B_{n}$, which were introduced in [10], are the total orderings arising from the natural action of $B_{n}$ on the open interval $] 0, \pi[$.

We briefly recall the definition. Let $D_{n}$ denote the unit disk, with $n$ punctures lined up on the horizontal diameter. We can equip $D_{n}$ with a hyperbolic structure (in which the punctures correspond to cusps). Indeed, many such hyperbolic structures exist, but we fix one choice, for the rest of the paper. 
A Nielsen-Thurston ordering is, by definition, induced by a finite or infinite geodesic ray $\gamma:[0, T] \rightarrow D_{n}$ (with $T \in \mathbb{R}_{+}$) or $\gamma:\left[0, \infty\left[\rightarrow D_{n}\right.\right.$, starting at a basepoint on the boundary of $D_{n}$, and (in the case of a finite ray) terminating at some other point of $\partial D_{n}$.

The ordering induced by $\gamma$ is defined as follows. Any geodesic starting on the basepoint in $\partial D_{n}$ has in its starting point a certain angle, belonging to the interval ] $0, \pi\left[\right.$, with the boundary $\partial D_{n}$. Conversely, the geodesic is uniquely determined by the value of this angle. More generally, even a non-geodesic ray starting on the basepoint is homotopic, relative to its end points, to a unique geodesic, so homotopy classes of such rays are still in one-to-one correspondence with angles in $] 0, \pi[$. Now the braid group, viewed as the mapping class group of $D_{n}$, acts on the geodesic $\gamma$; this yields a partial ordering $\prec$ of $B_{n}$, by defining that $\beta_{1} \prec \beta_{2}$ if and only if the angle of $\beta_{1} \cdot \gamma$ is smaller than the angle $\beta_{2} \cdot \gamma$.

If $\gamma$ is not invariant under the action of any braid, then this recipe determines a total ordering of $B_{n}$, and this ordering is called the Nielsen-Thurston ordering induced by $\gamma$. Notice that a necessary (but not sufficient) condition for $\gamma$ not being invariant under any braid action is that $\gamma$ cuts up the surface, in the sense that no two punctures are in the same path components of $\left.D_{n}-\gamma([0, T])\right)$.

An important property of Nielsen-Thurston orderings is the so-called subwordproperty, or property $S$. This means that for any two braid words $w$ and $w^{\prime}$, where $w^{\prime}$ is obtained from $w$ by inserting positive generators $\sigma_{i}$, we have : $w \prec w^{\prime}$. Geometrically, this is nothing but the fact that the left half-twist along any arc connecting two punctures pushes any curve intersecting the arc further to the left. In particular this half-twist is a positive element for the ordering.

The properties of the Nielsen-Thurston orderings are closely related to the geometric nature of the corresponding geodesic. Let us consider, for a maximal $m \in\{0,1, \ldots, n-1\}$, the $m$ points in time such that some pair of punctures is in the same path component of $D_{n}-\gamma\left(\left[0, t_{i}-\epsilon\right]\right)$, but in separate path components of $D_{n}-\gamma\left(\left[0, t_{i}\right]\right)$. We shall call this the sequence of separating moments.

If $m=n-1$, i.e. if a finite initial segment of $\gamma$ is sufficient to cut up the surface, then the ordering is said to be of finite type. If $m<n-1$ then the ordering is of infinite type. If $m=0$, i.e. if $\gamma$ has no self-intersections and no finite initial segment of $\gamma$ separates any pair of punctures, then we say the ordering is of full infinite type.

\section{Convex subgroups of Nielsen-Thurston orderings of Finite type}

Theorem 3.1. Suppose $\prec$ is a Nielsen-Thurston ordering of finite type, associated to a geodesic $\gamma$. Let $0<t_{1}<t_{2}<\ldots<t_{n-1}$ be the sequence of separating moments of $\gamma$. Then the chain of convex subgroups is

$$
\{1\} \subset G_{n-1} \subset \ldots \subset G_{1} \subset B_{n}
$$

where $G_{i}$ denotes the subgroup of $B_{n}$ consisting of all braids which preserve the geodesic segment $\gamma\left(\left[0, t_{i}\right]\right)$.

Proof. It is immediate that the subgroups thus described are convex, we only have to prove that they are the only ones. 
The proof is by downwards induction. The start of the induction is with the cases where all components of $D_{n}-\gamma\left(\left[0, t_{n-i}\right]\right)$ contain at most two punctures. In these cases, the subgroup consisting of elements of $B_{n}$ leaving $\gamma_{n-i}$ invariant is isomorphic to $\mathbb{Z}^{i}$, and is lexicographically ordered. Such an ordered group has no convex subgroups except the ones obtained by successively removing the largest generator - and these subgroups do indeed appear in the chain of convex subgroups $G_{n-1} \subset$ $\ldots \subset G_{n-i}$.

Let us now suppose inductively that for some index $i$ there are no convex subgroups in $G_{n-i}$ except $G_{n-j}$ with $j<i$. Let $G^{\prime}$ be a convex subgroup of $G_{n-i-1}$ such that $G_{n-i} \subsetneq G^{\prime} \subset G_{n-i-1}$. Our aim is to prove that $G^{\prime}=G_{n-i-1}$.

Let us recall the structure of the group $G_{n-i-1}$ : the geodesic $\gamma\left(\left[0, t_{n-i-1}\right]\right)$ cuts $D_{n}$ into a number of components, some of which contain punctures. If we denote $n_{1}, \ldots, n_{k}$ the number of punctures contained in each of these path components, then the subgroup $G_{n-i-1}$ is isomorphic to a product $G_{n-i-1} \cong B_{n_{1}} \times \ldots \times B_{n_{k}}$. Moreover, there is exactly one connected component of $D_{n}-\gamma\left(\left[0, t_{n-i-1}\right]\right)$ which is further cut by $\gamma\left(\left[t_{n-i-1}, t_{n-i}\right]\right)$ into pieces one of which contains $\lambda$ punctures and another one $\mu$ punctures (with $\lambda, \mu \geqslant 1$, and at least one of the two being $\geqslant 2$ ). Without loss of generality let us say the component being cut is the first one, so that $n_{1}=\lambda+\mu$.

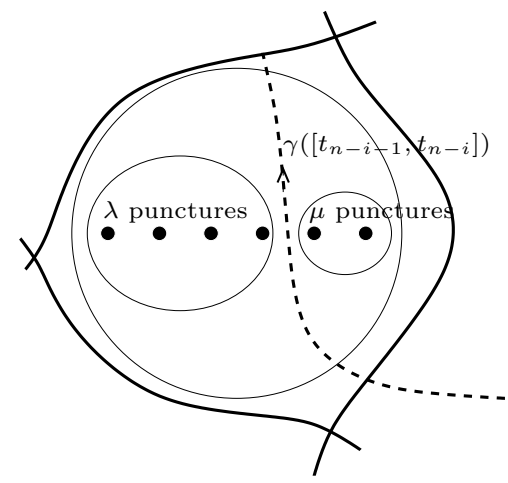

Figure 1. Fat lines: $\gamma\left(\left[0, t_{n-i-1}\right]\right)$. Dashed line: $\gamma\left(\left[t_{n-i-1}, t_{n-i}\right]\right)$.

Now there are three disks with geodesic boundary contained in the region under discussion: one containing $\lambda$ punctures, one containing $\mu$ punctures, and one containing both sets of punctures. Possibly after a conjugation we can assume that the three disks are round (i.e. their boundaries intersect the horizontal diameter only twice, see Figure 11).

We shall study the action of the hypothetical subgroup $G^{\prime}$ on the geodesic $\gamma\left(\left[0, t_{n-i}\right]\right)$, keeping in mind that it fixes the initial segment $\gamma\left(\left[0, t_{n-i-1}\right]\right)$. Let

$$
\gamma_{\max }=\sup _{g^{\prime} \in G^{\prime}} g^{\prime} \cdot \gamma
$$

(where the supremum is in the sense that every geodesic corresponds to a point of the real line, and we're taking the supremum of real numbers). What does the first intersection of $\gamma_{\max }$ with our critical component of $D_{n}-\gamma\left(\left[0, t_{n-i-1}\right]\right)$ look like? Notice that $\gamma_{\max }$ must be $G^{\prime}$-invariant. 


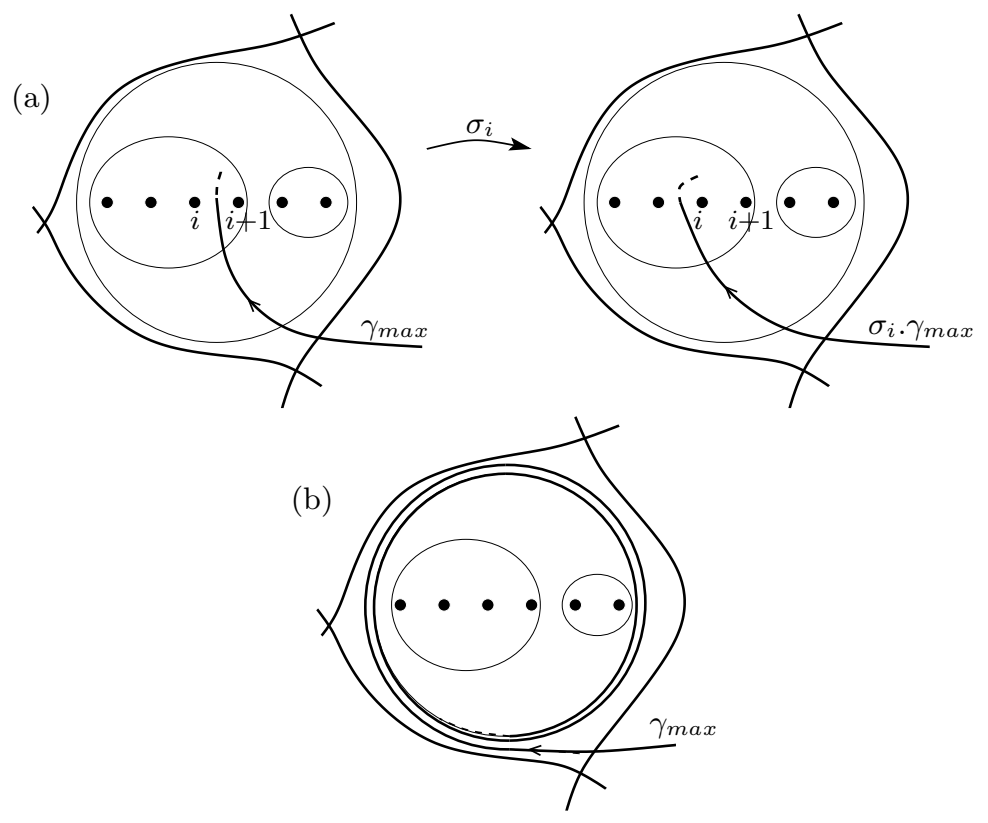

Figure 2.

The geodesic segment $\gamma_{\max }$ cannot pass between two punctures among the $\lambda$ punctures of the left disk, nor can it pass between two of the $\mu$ punctures of the right disk, for if it did, then the action of an appropriate element in the smaller convex subgroup $B_{\lambda}$ or $B_{\mu}$ would displace the geodesic even further to the left, which is excluded by construction (see Figure 2(a)). Suppose, on the other hand, that $\gamma_{\max }$ avoids entirely the two discs with geodesic boundary containing the set of $\lambda$ or $\mu$ punctures, but intersects the horizontal segment between the two disks. Then we have $g^{\prime} \cdot \gamma_{\max }>\gamma_{\max }$ or $\left(g^{\prime}\right)^{-1} \cdot \gamma_{\max }>\gamma_{\max }$, where $g^{\prime}$ is any element of $G^{\prime}$ which moves the segment $\gamma\left(\left[t_{n-i-1}, t_{n-i}\right]\right.$ ) (such an element must exist, since $G_{n-i} \subsetneq G^{\prime}$ ). This is also impossible.

In summary, the geodesic $\gamma_{\max }$ remains disjoint from the disk with geodesic boundary containing the $\lambda+\mu$ punctures; thus it spirals onto its boundary, as indicated in Figure 2(b). But this means that $g^{\prime} \cdot \gamma$ can get as big (far to the left) as any geodesic $g . \gamma$ with $g \in G_{n-i-1}$. This implies that $G^{\prime}=G_{n-i-1}$.

\section{Convex subgroups of Nielsen-Thurston orderings of full infinite} TYPE

We recall that a geodesic ray $\gamma$ in $D_{n}$ gives rise to an ordering of full infinite type if it starts at the basepoint in $\partial D_{n}$, has no self-intersections, separates the punctures, and is not stabilized by any nontrivial element of $B_{n}$.

The next result should be compared with [. 
Theorem 4.1. Suppose $\gamma$ is an infinite geodesic ray in $D_{n}$ giving rise to a NielsenThurston ordering $\prec$ of $B_{n}$ of full infinite type. Then $\prec$ has no convex subgroups except $\{1\}$ and $B_{n}$.

Proof. The strategy is to prove that $B_{n}$ can be generated by elements which are "arbitrarily close to the identity", which implies that any nontrivial convex subgroup contains all of $B_{n}$.

Here are the details. The geodesic $\gamma$ can be specified by an infinite word, where the $k$ th letter $(k \in \mathbb{N})$ specifies between which pair of punctures of $D_{n}$ the $k$ th intersection of $\gamma$ with the horizontal diameter occurs, and in which direction (up or down).

We shall prove that the braid group can be generated by $n-1$ elements $\tau_{1}, \ldots, \tau_{n-1}$ which all leave an arbitrarily long initial segment of $\gamma$ invariant: the words describing $\tau_{i} \cdot \gamma$ all coincide with the word describing $\gamma$ on an arbitrarily long initial segment.

Here is the construction of the elements $\tau_{i}$ : we trace out the geodesic $\gamma$, for at least the whole required initial segment, and continue until we pass close to a puncture (so close that the line we have drawn so far does not intersect the short segment from our current position to the puncture). At that moment, we deviate from the geodesic $\gamma$, and drop into the puncture. We replace the curve we have just drawn by a geodesic which is homotopic to it relative to its endpoints, and call this geodesic $\gamma^{\prime}$. The puncture at its end will be called the central puncture. Let $k$ be the number of intersections of $\gamma^{\prime}$ with the horizontal diameter (not counting the final drop into the central puncture).

There are geodesic arcs $a_{1}, \ldots, a_{n-1}$ disjoint from $\gamma^{\prime}$ and from each other, connecting the central puncture to the $n-1$ other punctures. For later use, we shall equip these arcs with an orientation pointing towards the central puncture. The braid $\tau_{i}$ will be a positive or negative half Dehn-twist around the $\operatorname{arc} a_{i}$ - the sign remains to be specified, depending on the relative position of terminal segments of $\gamma^{\prime}$ and $a_{i}$.

We shall consider three cases. Firstly, if the last intersections of these two arcs with the horizontal diameter do not occur between the same pairs of punctures, then the sign of the half Dehn twist can be chosen arbitrarily. Indeed, in this case the first $k$ intersections of $\tau_{i} \cdot \gamma$ with the horizontal diameter coincide with those of $\gamma$, independently of whether $\tau_{i}$ is taken to be the positive or negative half twist along $a_{i}$ - see Figure 3(a).

The second case to consider is where the last intersections of the the arcs $\gamma^{\prime}$ and $a_{i}$ with the horizontal diameter lie between the same pairs of punctures, and where, moreover, the terminal segment of $a_{i}$ lies to the right of the terminal segment of $\gamma^{\prime}$ (as seen while approaching the central puncture along $\gamma^{\prime}$ ) - see Figure 3(b) for examples. In this case, we take $\tau_{i}$ to be the positive half twist along $a_{i}$. Again, we observe that with this choice the word describing the first $k$ intersections of $\tau_{i} \cdot \gamma$ with the horizontal diameter coincides with the corresponding word for $\gamma$.

The third and final case is where the last intersections of the the $\operatorname{arcs} \gamma^{\prime}$ and $a_{i}$ with the horizontal diameter lie between the same pairs of punctures, and where 
(a)

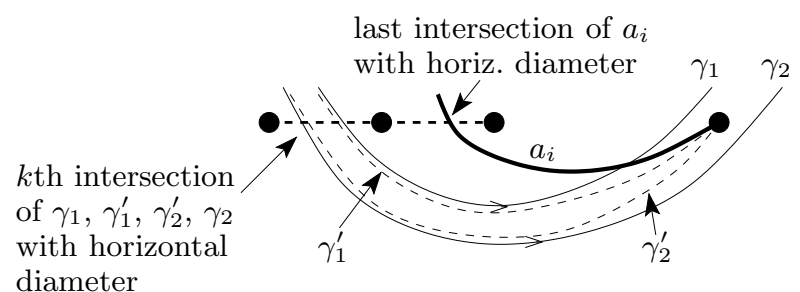

(b)

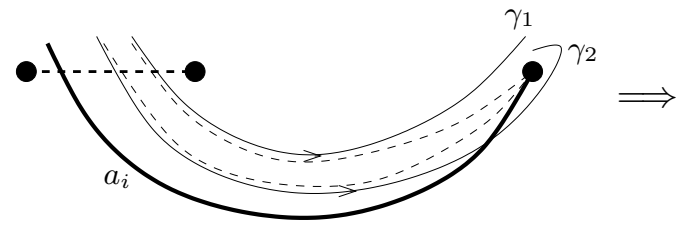

perform positive half Dehn twist along $a_{i}$

(c)

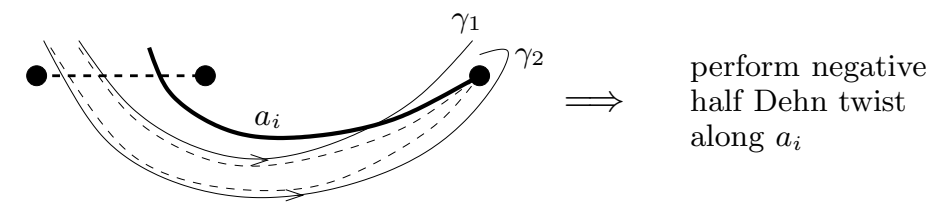

Figure 3.

the terminal segment of $a_{i}$ lies to the left of the terminal segment of $\gamma^{\prime}$. In this case, we take $\tau_{i}$ to be the negative half twist along $a_{i}-$ see Figure $3(\mathrm{c})$.

\section{Convex subgroups of General Nielsen-Thurston orderings}

By combining the techniques of proof of Theorems 3.1 and 4.1, one can easily obtain the following unified result:

Theorem 5.1. Suppose $\prec$ is a Nielsen-Thurston ordering, associated to a geodesic $\gamma$. Let $0<t_{1}<t_{2}<\ldots<t_{m}$, with $m \in\{0,1, \ldots, n-1\}$, be the sequence of separating moments of $\gamma$. Then the chain of convex subgroups is

$$
\{1\} \subset G_{m} \subset \ldots \subset G_{1} \subset B_{n}
$$

where $G_{i}$ denotes the subgroup of $B_{n}$ consisting of all braids which preserve the geodesic segment $\gamma\left(\left[0, t_{i}\right]\right)$.

\section{The Conradian soul of Nielsen-Thurston orderings}

Recall that a group ordering $\prec$ is said to be Conradian if for every positive $f, g$

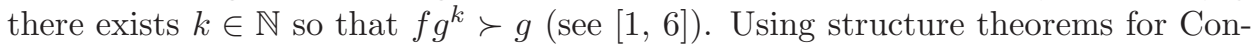
rad orderable groups and the description of the convex subgroups from the previous sections, one can easily show that no Nielsen-Thurston ordering is Conradian (c.f. Corollary 6.4). However, we prefer giving a longer proof which avoids these structure theorems. In this proof, the cases of finite type orderings on $B_{3}$ and $B_{4}$ need a special argument (compare Remark 6.5).

Example 6.1. The Dehornoy ordering $\prec_{D}$ on $B_{3}$ is not Conradian. This is shown in 8, Example 3.21], through a very indirect argument. A shorter proof works 
as follows: using the identity $\sigma_{2} \sigma_{1} \sigma_{2}^{-1}=\sigma_{1}^{-1} \sigma_{2} \sigma_{1}$ we get, for all $k \in \mathbb{N}$, that $1 \prec_{D} \sigma_{2} \sigma_{1} \sigma_{2}^{-(k+1)}=\sigma_{1}^{-(k+1)} \sigma_{2} \sigma_{1}$. Therefore, $\left(\sigma_{2}^{-1} \sigma_{1}\right)\left(\sigma_{1}^{k}\right) \prec_{D} \sigma_{1}$ for all $k \in \mathbb{N}$, yet both $\sigma_{2}^{-1} \sigma_{1}$ and $\sigma_{1}$ are $\prec_{D}$-positive.

Notice that, according to [10], every Nielsen-Thurston ordering of finite type on $B_{3}$ is conjugate to the Dehornoy ordering. Since the Conrad property is stable under conjugacy, no Nielsen-Thurston ordering of finite type on $B_{3}$ is Conradian.

Example 6.2. Up to conjugacy, there are only three Nielsen-Thurston orderings of finite type on $B_{4}$, namely those given by the geodesics of Figure 4 below (see again 10). None of them gives rise to a Conradian ordering. Indeed, (a) corresponds to the Dehornoy ordering, and the above argument applies. For the geodesics in (b) and (c), one can directly check the inequality $1 \prec \sigma_{3} \sigma_{2} \sigma_{3}^{-(k+1)}$, which allows to conclude as in the previous example.
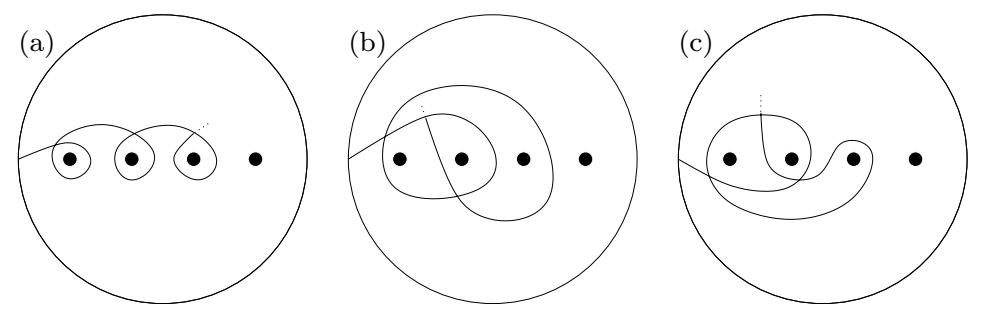

Figure 4. The three conjugacy classes of finite type NielsenThurston orderings of $B_{4}$.

Following [8, 9], the Conradian soul of an ordering is defined as the maximal convex subgroup restricted to which the order satisfies the Conrad property. It was already shown in 8 that the Conradian soul of the Dehornoy ordering on $B_{n}$ corresponds to $\left\langle\sigma_{n-1}\right\rangle$. Next, we describe the Conradian soul of any Nielsen-Thurston ordering, thus solving a problem raised in [8, Remark 3.40].

Theorem 6.3. The Conradian soul of a Nielsen-Thurston ordering on $B_{n}$ corresponds to the maximal Abelian convex subgroup, that is, to the largest copy of $B_{2} \times \cdots \times B_{2}$ which is convex.

Proof. For a geodesic of infinite type, the smallest non-trivial convex subgroup is a copy of $B_{m}$ for some $m$ with $3 \leqslant m \leqslant n$. Such a group has no convex subgroup. If it was Conradian, then it would be Archimedean, and hence by Hölder's theorem, the underlying group would be Abelian (see [1, 6]). This is absurd as $B_{m}$, for $m \geqslant 3$, is not Abelian.

If the geodesic is of finite type, then it is clear that the maximal convex copy of a product of (say, $k$ ) $B_{2}$ factors is contained in the Conradian soul (indeed, any bi-invariant ordering - and thus any ordering defined on an Abelian group is Conradian). The next larger convex subgroup of $B_{n}$ corresponds either to a product $P=B_{n_{1}} \times \cdots \times B_{n_{k-1}}$, where there is only one index different from 2, which is equal to 4 , or to a product $P=B_{n_{1}} \times \cdots \times B_{n_{k}}$, where only one index is different from 2 , and it is equal to 3 . If the restriction of the ordering to $P$ was 
Conradian, then the restriction to $B_{4}$ or $B_{3}$, respectively, would be also Conradian. However, we have seen in Examples 6.1 and 6.2 that this cannot be the case.

An alternative argument for the end of the proof. To see that the restriction of the ordering to $P$ cannot be Conradian, first note that $P$ has only finitely many convex subgroups. By [9, Proposition 1.7] and its proof, if $P$ was Conradian then the underlying group would be solvable. However, neither $B_{3}$ nor $B_{4}$ are solvable.

\section{Corollary 6.4. No Nielsen-Thurston ordering is Conradian.}

Proof. If a Nielsen-Thurston ordering was Conradian, then it would coincide with its Conradian soul. However, according to Theorem 6.3, this is never the case.

Remark 6.5. Although stated for Nielsen-Thurston orderings on general braid groups $B_{n}$ (with $n \geqslant 3$ ), the preceding corollary gives new information only in the cases of $B_{3}$ and $B_{4}$. Indeed, these groups do admit Conradian orderings, whereas for $n \geqslant 5$ there is no Conradian ordering on $B_{n}$ at all (see [2, pp. 287-289]).

\section{Nielsen-Thurston ORderings of infinite type ARE APPROXimated By THEIR CONJUGATES}

The relevance of the Conradian soul stems from the following result which we cite from [8, Proposition 4.7] (see also [4, Theorem 1.2] and 9, Theorem 2.7]).

Theorem 7.1. If the Conradian soul of an ordering on an infinite countable group is trivial, then this ordering is an accumulation point of its conjugates.

Using this result we may easily prove the following

Theorem 7.2. Any Nielsen-Thurston ordering of infinite type on $B_{n}$ may be approximated by its conjugates.

Proof. By Theorem 6.3, the Conradian soul of the ordering is trivial. The result then follows from Theorem 7.1.

\section{Nielsen-ThURSTON ORDERINGS OF FINITE TYPE ARE NOT ISOlATED}

We now show our main result.

Theorem 8.1. Nielsen-Thurston orderings are not isolated in the topological space of left-invariant orderings of $B_{n}$.

Proof. In the case of orderings of infinite type, the ordering may be approximated by its conjugates (see Theorem 7.2).

In the finite type case, for a maximal value of $k$ there exists a convex subgroup of the form $B_{2} \times \cdots \times B_{2} \sim \mathbb{Z}^{k}$. If $k \geqslant 2$, then the restriction of $\prec$ to $\mathbb{Z}^{k}$ is not isolated in the space of orderings of $\mathbb{Z}^{k}$ (see for instance [11]). A convex extension argument (see $[$, Section 3.3.5]) then shows that $\prec$ is not isolated in the space of 
braid orderings. If $k=1$, then the smallest convex subgroup strictly containing the maximal Abelian convex subgroup $B_{2}$ is a copy of $B_{3}$. By [10], the restriction of $\prec$ to this copy of $B_{3}$ is a conjugate of the Dehornoy ordering, which is not isolated in the space of orderings of $B_{3}$ : see Example 8.2 below. Once again, a convex extension argument shows that $\prec$ is not isolated in the space of braid orderings.

Example 8.2. Dehornoy's ordering is approximated by its conjugates. This was first shown in 8] throughout a very indirect argument. A simpler proof appears in 2. Chapter XIV]. Here we propose an even simpler argument.

Let $\prec_{j}$ be the Dehornoy ordering, conjugated by $\sigma_{2}^{-j} \sigma_{1}$. Thus, a word $w$ is positive in the ordering $\prec_{j}$ if and only if $\sigma_{1}^{-1} \sigma_{2}^{j} w \sigma_{2}^{-j} \sigma_{1} \succ_{D} 1$. We claim that the sequence $\prec_{j}$ tends to $\prec_{D}$ in the space of orderings.

Indeed, if $w=\sigma_{2}^{k}$ for some $k>0$, then $\sigma_{1}^{-1} \sigma_{2}^{j} w \sigma_{2}^{-j} \sigma_{1} \succ_{D} 1$ since the Dehornoy order has Property S. If, on the other hand, $w$ is a $\sigma_{1}$-positive word: $w=\sigma_{2}^{k_{1}} \sigma_{1} \sigma_{2}^{k_{2}} \sigma_{1} \ldots \sigma_{2}^{k_{\ell-1}} \sigma_{1} \sigma_{2}^{k_{\ell}}$, then we calculate

$$
\begin{aligned}
\sigma_{1}^{-1} \sigma_{2}^{j} w \sigma_{2}^{-j} \sigma_{1} & =\sigma_{1}^{-1} \sigma_{2}^{j} \sigma_{2}^{k_{1}} \sigma_{1} \sigma_{2}^{k_{2}} \sigma_{1} \ldots \sigma_{2}^{k_{\ell-1}} \sigma_{1} \sigma_{2}^{k_{\ell}} \sigma_{2}^{-i} \sigma_{1} \\
& =\sigma_{2} \sigma_{1}^{j+k_{1}} \sigma_{2}^{-1} \sigma_{2}^{k_{2}} \sigma_{1} \ldots \sigma_{2}^{k_{\ell-1}} \sigma_{1} \sigma_{2}^{k_{\ell}} \sigma_{2}^{-n} \sigma_{1} .
\end{aligned}
$$

Thus $\sigma_{1} \sigma_{2}^{-j} w \sigma_{2}^{j} \sigma_{1}$ is $\sigma_{1}$-positive for sufficiently large $j$ (namely for $j>-k_{1}$ ), which proves the desired convergence.

Finally, $\prec_{j}$ is different from $\prec_{D}$ for all positive integers $j$, since its smallest positive element is the conjugate of $\sigma_{2}$ by $\sigma_{2}^{-j} \sigma_{1}$, and this is different from $\sigma_{2}$.

Remark 8.3. The choice of the sequence $\sigma_{2}^{-j} \sigma_{1}$ is quite natural. Indeed, the sequence $\sigma_{2}^{-j} \sigma_{1}$ approaches the Conradian soul from above: for any element $c$ of the Conradian soul $\left\langle\sigma_{2}\right\rangle$ and for any $\sigma_{1}$-positive word $w$ we have $c \prec_{D} \sigma_{2}^{-j} \sigma_{1} \prec_{D} w$, where the second inequality is only true for sufficiently large $j$. In terms of geodesics, if $\gamma$ is the standard geodesic inducing the Dehornoy-order on $B_{3}$ (c.f. Figure $4(\mathrm{a})$ ), then the sequence of geodesics $\sigma_{2}^{-j} \sigma_{1} \cdot \gamma$ is eventually further to the right than any prescribed geodesic $\beta . \gamma$, for $\beta$ a $\sigma_{1}$-positive braid - see Figure 5 .

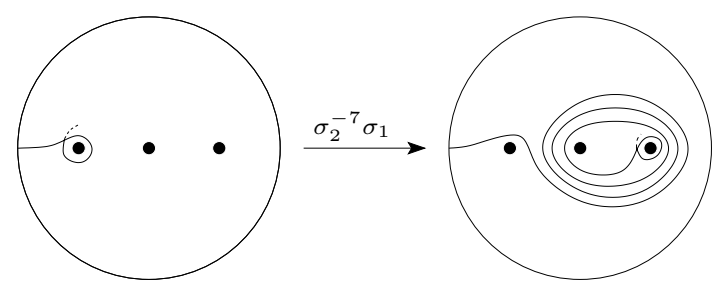

Figure 5. The geodesic $\sigma_{2}^{-j} \sigma_{1} \cdot \gamma$ deviates from $\gamma$ to the left, but "by as little as possible": it goes "over" the leftmost puncture (thus deviating to the left), but then it goes as much to the right as possible (it turns $j$ times counterclockwise around the two rightmost punctures, with $j$ arbitrarily large)

Example 8.2 can be generalized as follows. 
Theorem 8.4. Every Nielsen-Thurston ordering $\prec$ of finite type whose Conradian soul is a copy of $B_{2}$ can be approximated by its conjugates.

Proof. If $\sigma$ is a positive generator of the Conradian soul, then $\sigma$ corresponds to a left half-twist. According to [8, Proposition 4.9] and its proof (see also [9, Theorem 2.9] and its proof), there exists a sequence $\prec_{j}=g_{j}(\prec)$ of conjugates of $\prec$ which converges either to $\prec$ or to $\prec^{\prime}$, where $\prec^{\prime}$ is the ordering whose set of positive elements is the union of $\left\{\sigma^{k}: k<0\right\}$ and $\{g: g \succ 1, g \notin\langle\sigma\rangle\}$. If the limit point was $\prec^{\prime}$, then the conjugates $g_{j}^{-1} \sigma g_{j}$ would be negative for $j$ large enough. This contradicts the fact that the Nielsen-Thurston ordering $\prec$ has the subword property $\mathrm{S}$.

Remark 8.5. The argument of the proof above actually shows the stronger result that for any given Nielsen-Thurston ordering of finite type $\prec$, there exists another finite-type Nielsen-Thurston ordering $\prec_{1}$, obtained from the given one simply by permuting the lexicographic order of the Conradian soul, which is approximated by conjugates of itself (compare [8, Proposition 4.9] and [9, Corollary 2.10]).

The previous results show that the only Nielsen-Thurston orderings which may fail to be approximated by their conjugates are those of finite type whose Conradian soul is higher-rank Abelian.

Example 8.6. Consider the ordering $\prec$ induced by the geodesic shown in Figure 6(a). The Conradian soul of this ordering is $\left\langle\sigma_{1}, \sigma_{3}, \sigma_{5}\right\rangle=\mathbb{Z}^{3}$. By Theorem 8.1. this order can be approximated by other orders, but we do not know whether it can be approximated by its conjugates. Imitating Example 8.2 and the proof of Theorem 8.4, we could try to conjugate the order by elements which are as small as possible while being larger than all elements of the Conradian soul. This, however, yields an approximation not of the desired ordering $\prec$, but of one where the lexicographic order of $\mathbb{Z}^{3}$ has been permuted.

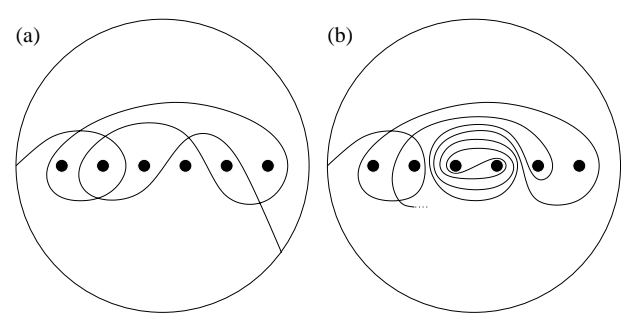

Figure 6. (a) A geodesic whose associated ordering of $B_{6}$ seems not to be a limit point of Nielsen-Thurston orderings. Note the convex subgroups $\left\langle\sigma_{5}\right\rangle \subseteq\left\langle\sigma_{3}, \sigma_{5}\right\rangle \subseteq\left\langle\sigma_{1}, \sigma_{3}, \sigma_{5}\right\rangle$. (b) Conjugating by $\sigma_{3}^{-N} \sigma_{4}$ (a sequence which becomes eventually smaller than any positive element outside the Conradian soul) yields an approximation of a different ordering with convex jumps $\left\langle\sigma_{1}\right\rangle \subseteq\left\langle\sigma_{1}, \sigma_{5}\right\rangle \subseteq$ $\left\langle\sigma_{1}, \sigma_{3}, \sigma_{5}\right\rangle$. 
Question. Can the ordering induced by the geodesic of Figure 5(a) be approximated by its conjugates? If not, can it be approximated by a sequence of NielsenThurston orderings? The calculation above suggests that the answer to both questions may be "no".

Acknowledgments. The first-named author was funded by PBCT-CONICYT Research Network on Low Dimensional Dynamics.

\section{REFERENCES}

[1] R. Botto-Mura \& A. Rhemtulla. Orderable groups. Lecture Notes in Pure and Applied Mathematics, Vol. 27. Marcel Dekker, New York-Basel (1977).

[2] P. Dehornoy, I. Dynnikov, D. Rolfsen, \& B. Wiest. Ordering Braids. Math. Surveys and Monographs 148 (2008).

[3] T. Dubrovina \& N. Dubrovin. On braid groups. Sbornik Mathematics 192 (2001), 693-703.

[4] A. Clay. Isolated points in the space of left-orderings of a group. Preprint (2009), arXiv:0812.2499.

[5] A. Clay. An exotic left ordering of the free group from the Dehornoy ordering. Preprint (2009).

[6] V. Kopitov \& N. Medvedev. Right ordered groups. Siberian School of Algebra and Logic, Plenum Publ. Corp., New York (1996).

[7] P. Linnell. The topology on the space of left orderings of a group. Preprint (2006), arXiv:math/0607470.

[8] A. Navas. On the dynamics of left-orderable groups. Preprint (2007), arXiv:0710.2466.

[9] A. Navas \& C. Rivas, with an appendix by A. Clay. A new characterization of Conrad's property for group orderings, with applications. Preprint (2009), arXiv:0901.0880.

[10] H. Short \& B. WiEst. Orderings of mapping class groups after Thurston. L'Enseignement Mathématique 46 (2000), 279-312.

[11] A. Sikora. Topology on the spaces of orderings of groups. Bull. London Math. Soc. 36 (2004), 519-526.

Andrés Navas, Univ. de Santiago de Chile, Alameda 3363, Santiago, Chile

E-mail address: andres.navas@usach.cl

Bert Wiest, IRMAR (UMR 6625 du CNRS), Université de Rennes 1, Campus de Beaulieu, 35042 Rennes Cedex, France

E-mail address: bertold.wiest@univ-rennes1.fr 